\section{Indicadores de saúde materno infantil em Belo Horizonte, Minas Gerais, Brasil, 2001: análise dos diferenciais intra-urbanos}

\author{
Maternal and child health indicators in \\ Belo Horizonte, Minas Gerais State, Brazil, 2001: \\ an analysis of intra-urban differences
}

\author{
1 Secretaria Municipal \\ de Saúde de Belo Horizonte, \\ Belo Horizonte, Brasil. \\ 2 Faculdade de Medicina, \\ Universidade Federal \\ de Minas Gerais, \\ Belo Horizonte, Brasil. \\ 3 Instituto de Ciências \\ Exatas, Universidade \\ Federal de Minas Gerais, \\ Belo Horizonte, Brasil. \\ Correspondência \\ A. A. L. Friche \\ Secretaria Municipal de \\ Saúde de Belo Horizonte. \\ Rua Grão Mogol 815 , \\ apt. 301 B, Belo Horizonte, $M G$ \\ 30110-010, Brasil. \\ gutafriche@uol.com.br \\ gutafriche@gmail.com
}

\begin{abstract}
Spatial analysis of health indicators is as an important methodology for detection of intra-urban differences. This study aimed to examine the spatial distribution of all live births in Belo Horizonte, analyzing the presence of spatial clusters of health indicators for newborns and their mothers, using data from the Information System on Live Births. For each area covered by a Primary Health Care Unit, we calculated the indicators using empirical Bayesian methods. For spatial analysis, the indicators obtained from the global Moran (I) index and Local Indicators of Spatial Association (LISA) were used. Analysis using LISA showed the presence of relevant spatial clusters for adolescent mothers and those with low schooling, stillbirths in previous pregnancies, cesarean sections, and low attendance at prenatal care, especially in areas with low socio-demographic characteristics. The methodology adopted was configured as a key instrument for detecting risk areas where clustering occurs. The method can easily be incorporated into health surveillance systems as a mechanism for controlling events related to births in a given area.
\end{abstract}

Spatial Analysis; Health Status Indicators; Maternal and Child Health; Social Conditions
Amélia Augusta de Lima Friche 1,2

Waleska Teixeira Caiaffa 2

Cibele Comini César 3

Lúcia Maria de Figueiredo Goulart ${ }^{2}$

Maria Cristina de Mattos Almeida 1

\section{Introdução}

O conhecimento de determinantes de saúde de uma população é de fundamental importância para o planejamento e a implementação de estratégias efetivas de melhoria das condições de saúde. Especialmente, a identificação dos determinantes da morbimortalidade tem contribuído significativamente para elaboração de planejamento e assistência adequada. Vários estudos têm abordado o perfil de natalidade e mortalidade da população materno infantil, considerando-se fatores de risco como peso ao nascimento, duração da gestação e condições sócioeconômicas 1,2,3,4,5,6,7,8. Entretanto, esses determinantes ainda são pouco explorados no que se refere à contextualização geográfica espacial.

A análise de indicadores de saúde sob a perspectiva espacial configura-se como instrumento importante na detecção de diferenciais intra-urbanos, subsidiando ações de planejamento, organização e execução das ações de saúde de determinada região 9,10,11. As técnicas de mapeamentos e análises espaciais, particularmente de ambientes denominados Sistemas de Informações Geográficas (GIS), têm possibilitado uma melhor compreensão do processo saúde-doença na população, além da descoberta de padrões espaciais e relações entre os seus componentes, que incluem não só as características da divisão territorial, mas também aquelas relacionadas ao ambiente físico, proxi- 
midade e qualidade dos serviços de saúde e redes sociais das áreas de estudo 12,13,14. Experiências usando essas técnicas têm se acumulado nas áreas de mortalidade, doenças crônicas e de notificação compulsória 15,16,17,18. Previamente, utilizamos essas técnicas de análises para estabelecer áreas de alto risco para doenças endêmicas, epidêmicas e eventos sob vigilância epidemiológica usados para planejar, implementar e avaliar intervenções de saúde pública em áreas urbanas e periurbanas 19,20,21.

No Brasil, com a implantação e aprimoramento constante do Sistema de Informação sobre Nascidos Vivos (SINASC) a partir de 1990 e, em Belo Horizonte, Minas Gerais, desde 1991, vários estudos têm sido realizados com essas informações, objetivando conhecer o perfil da morbimortalidade da população materno infantil através da construção de indicadores de saúde 2,4,6. Com a implantação definitiva do sistema em todas as maternidades do Município de Belo Horizonte, em 1992, tornou-se possível a realização de investigações sistemáticas das informações do SINASC. Associa-se a essa implantação o conhecimento recente sobre a qualidade de informação desse sistema. Em estudo realizado, comparando-se as informações obtidas em prontuários hospitalares e algumas informações constantes no SINASC da cidade de Belo Horizonte, foi encontrada excelente qualidade e confiabilidade da informação 22.

O presente estudo objetiva, a partir dos dados do SINASC, traçar um perfil dos nascimentos em Belo Horizonte, no ano de 2001, sob a hipótese de que a distribuição espacial de alguns indicadores de saúde, relacionados às mães e aos recém-nascidos, não ocorre de forma aleatória, mas produzindo conglomerados de áreas com autocorrelação espacial significativa, contribuindo para subsidiar a organização e o planejamento das ações de vigilância à saúde da criança e da mulher no município.

\section{Material e métodos}

\section{Local do estudo e fonte dos dados}

O estudo foi realizado em Belo Horizonte, capital do Estado de Minas Gerais, com uma população total de 2.238.332 habitantes, a qual se distribui em uma área de $330,23 \mathrm{~km}^{2}$. O município tem notificado uma média de 35 mil nascimentos nos últimos 4 anos, com uma taxa de mortalidade infantil estimada de 14,1 por $1 \mathrm{mil}$ nascidos vivos em 2003.

O sistema de atendimento às gestantes inclui acesso e cobertura universal, e os dados analisados foram obtidos através do SINASCBH do ano de 2001, consolidados pela Gerência de Epidemiologia e Informação da Secretaria Municipal de Saúde de Belo Horizonte, acrescidos de sistema de informação complementar (COMPNASC), permitindo a sua identificação a partir do endereço da mãe. As variáveis do SINASC classificadas em características da mãe, da gestação e parto e do recém-nascido foram: idade da mãe; escolaridade; número de filhos em gestações anteriores nascidos vivos e mortos; duração da gestação; tipo de parto e número de consultas no pré-natal; índice de Apgar e peso ao nascer.

A organização territorial dos serviços de saúde do município encontrava-se, em 2001, subdividida em nove distritos sanitários, onde se distribuem 129 unidades básicas de saúde, cujas áreas de abrangência são estabelecidas com base nos 2.563 setores censitários definidos pelo Instituto Brasileiro de Geografia e Estatística IBGE - sendo constituídas por setores contíguos, respeitando-se os seus limites 23. A definição das áreas de abrangência foi feita a partir do processo de territorialização do município, em 1992, considerando o acesso da população aos serviços, suas barreiras geográficas e as malhas viárias. As áreas foram digitalizadas, passando a integrar a base geográfica do município 9 .

Os indicadores de saúde foram elaborados agregados por área de abrangência e subdivididos em indicadores da mãe (história reprodutiva, sua identificação e algumas de suas características), da gestação e parto e do recém-nascido. Para os primeiros, foram calculadas as proporções de mães adolescentes (idade $<20$ anos), com menos de oito anos de estudo, primíparas (nenhum filho em gestações anteriores), multíparas (com mais de três filhos vivos ou mortos em gestações anteriores) e com filhos nascidos mortos (pelo menos, um filho nascido morto em gestações anteriores). Para os segundos, isto é, relativos à gestação e parto, foram calculadas as proporções de cesáreas, menos de quatro consultas no pré-natal e de prematuros (idade gestacional $<37$ semanas). Sobre os recém-nascidos, foram calculadas as proporções de asfixia moderada no 1o e 5o minutos (Apgar $<7$ ), asfixia grave no 1o e 5 o minutos (Apgar $<4$ ), recém-nascido de baixo peso (peso ao nascer $<2.500 \mathrm{~g}$ ) e recém-nascido de muito baixo peso (peso ao nascer $<1.500 \mathrm{~g}$ ).

\section{Análise dos dados}

Foram construídos mapas cloropléticos da distribuição dos indicadores por área de abran- 
gência. Para minimizar as flutuações aleatórias devido ao reduzido número de recém-nascidos vivos em algumas áreas, foi utilizado o método Bayesiano empírico 24.

Para o estudo exploratório do padrão espacial, foram utilizados os índices de Moran global (I) e local (LISA). As proporções estimadas dos indicadores por área de abrangência foram obtidas através do método Bayesiano empírico 25,26,27. O índice global (I) fornece uma medida geral da associação espacial existente no conjunto de dados, medindo o grau de correlação espacial entre os pares de vizinhança, ponderado pela proximidade geográfica. $\mathrm{O}$ índice local é uma decomposição da medida global de autocorrelação espacial. Diferentemente do índice global, que produz um único valor para o conjunto de dados, os indicadores locais produzem um valor específico para cada área, permitindo a identificação de conglomerados de áreas com padrões significativos de associação espacial. Os valores da estatística de Moran, em geral, variam entre -1 e +1 , embora possam assumir valores diferenciados em alguns casos. Para a construção da matriz de vizinhança, foram considerados os vizinhos de primeira ordem, ou seja, as áreas de abrangência que compartilham fronteiras.

Para visualização das áreas com autocorrelação espacial estatisticamente significantes ( $\mathrm{p} \leq 0,05$ ), foram utilizados mapas representativos do índice de Moran local (LISA) para cada indicador estudado. Esses mapas possibilitaram a identificação de conglomerados de áreas com altas taxas do indicador e vizinhos com taxas relativamente elevadas (alto-alto) e áreas com taxas baixas do indicador e vizinhos com taxas relativamente baixas (baixo-baixo); além de áreas de transição, com taxas baixas e vizinhos com taxas altas (baixo-alto) ou taxas altas e vizinhos com taxas baixas (alto-baixo) 26,28.

Utilizou-se o coeficiente de correlação de Pearson (r) para avaliar a presença de associação linear entre as variáveis estudadas.

Para a análise dos dados, foram utilizados os programas SPSS for Windows (SPSS Inc., Chicago, Estados Unidos), MapInfo (MapInfo Corporation, New York, Estados Unidos) e GeoDa (Spatial Analysis Laboratory, University of Illinois, Urbana-Chanpaign, Estados Unidos).

\section{Resultados}

No ano de 2001, foram registrados, no SINASC, 36.127 nascimentos de residentes em Belo Horizonte. O percentual de informações ignoradas variou entre $0 \%$ para idade da mãe e $6,5 \%$ para número de filhos nascidos mortos em gestações anteriores.

Os indicadores, sua distribuição no município, suas médias e desvios padrões estimados pelo método Bayesiano empírico, além dos valores mínimos e máximos por áreas de abrangência, podem ser observados na Tabela 1. Em geral, as proporções observadas para o município foram bem próximas às proporções médias estimadas pelo método Bayesiano para as áreas de abrangência, com exceção dos indicadores de asfixia moderada no lo minuto, cuja média ponderada por áreas de abrangência foi menor que a observada. Quanto à variação entre áreas de abrangência, alguns indicadores variaram muito, tais como a proporção de mães adolescentes $(6,05 \%$ a $25,79 \%)$, menos de oito anos de estudo $(9,96 \%$ a $78,7 \%)$, primíparas $(31,78 \%$ a $50,35 \%)$ e cesárea $(27,89 \%$ a $67 \%)$. Outros indicadores apresentaram amplitudes menores, como a proporção de recém-nascidos com asfixia grave no 5 o minuto $(0,23 \%$ a $0,67 \%)$ e com muito baixo peso ( $1,11 \%$ a $2,06 \%)$.

A análise da estatística I de Moran global mostrou a presença de autocorrelação espacial positiva, com valores variando de 0,36 a 0,21 (todas com $\mathrm{p}=0,001$, após 999 permutações) para a proporção de mães adolescentes, com menos de oito anos de estudo, primíparas, com filhos mortos, cujo parto foi cesárea e cujo prénatal constou de menos de quatro consultas. Os indicadores referentes ao recém-nascido, tais como prematuridade, asfixia moderada e grave no 1o minuto e moderada no 5o minuto, além de muito baixo peso, apesar de apresentarem significância estatística, mostraram uma fraca autocorrelação espacial, com I variando entre 0,14 a 0,11 . A proporção de multíparas apresentou correlação baixa $(0,08)$, com significância limítrofe $(\mathrm{p}=0,06)$, e para os indicadores baixo peso e asfixia grave no 5o minuto, houve ausência de dependência espacial significativa, indicando uma distribuição aleatória desses indicadores no município.

A análise de autocorrelação espacial local (LISA) mostrou a presença de conglomerados de áreas com índices significativos para os indicadores mães adolescentes, menos de oito anos de estudo, primíparas, filhos mortos em gestações anteriores, cesárea e menos de quatro consultas no pré-natal. Os índices significantes ( $\mathrm{p} \leq 0,05)$, isto é, aqueles com proporções e vizinhos com taxas altas e os com proporções e vizinhos com taxas baixas podem ser visualizados nas Figuras 1 a 4 .

Na Figura 1, estão representados os conglomerados para mães adolescentes. Observa-se um conglomerado de taxas altas de mães me- 
Distribuição dos indicadores materno infantis segundo as áreas de abrangência ( $n=129)$. Belo Horizonte, Minas Gerais, Brasil, 2001.

\begin{tabular}{|c|c|c|c|c|c|}
\hline \multirow[t]{2}{*}{ Variáveis } & \multicolumn{5}{|c|}{ Proporção (\%)* } \\
\hline & Município & Média & Desvio padrão* & Mínima & Máxima \\
\hline Adolescente (idade, vinte anos) & 16,00 & 16,03 & 4,04 & 6,05 & 25,79 \\
\hline Menos de oito anos de estudo & 42,40 & 42,05 & 11,89 & 9,96 & 78,70 \\
\hline Primípara & 41,90 & 41,89 & 3,78 & 31,78 & 50,35 \\
\hline Multípara** & 5,10 & 5,12 & 1,57 & 2,05 & 11,42 \\
\hline Filho morto*** & 5,10 & 5,20 & 7,49 & 3,43 & 7,70 \\
\hline Cesárea & 43,60 & 43,57 & 8,23 & 27,89 & 67,00 \\
\hline Menos de quatro consultas no pré-natal & 9,90 & 9,86 & 2,85 & 3,98 & 17,76 \\
\hline Prematuridade\# & 7,70 & 7,73 & 0,54 & 6,14 & 8,89 \\
\hline Asfixia moderada 1ㅇ minuto (Apgar $<7$ ) & 14,00 & 8,15 & 0,42 & 7,16 & 9,33 \\
\hline Asfixia grave 1 o minuto (Apgar $<4$ ) & 2,60 & 2,59 & 0,19 & 2,13 & 3,08 \\
\hline Asfixia moderada 5 ㅇ minuto (Apgar < 7) & 2,80 & 1,37 & 0,14 & 1,08 & 1,85 \\
\hline Asfixia grave 5 으 minuto (Apgar $<4)$ & 0,40 & 0,35 & 0,08 & 0,23 & 0,67 \\
\hline Baixo peso (peso ao nascer $<2.500 \mathrm{~g}$ ) & 10,60 & 10,58 & 0,33 & 9,81 & 11,50 \\
\hline Muito baixo peso (peso ao nascer $<1.500 \mathrm{~g}$ ) & 1,50 & 1,52 & 0,20 & 1,11 & 2,06 \\
\hline
\end{tabular}

* Estimativa Bayesiana empírica por área de abrangência;

** Mais de quatro filhos tidos em gestações anteriores nascidos vivos ou mortos;

*** Um ou mais filhos tidos em gestações anteriores nascidos mortos;

\# Idade gestacional < 37 semanas.

Fonte: SINASC-Belo Horizonte. Gerência de Epidemiologia e Informação,

Secretaria Municipal de Saúde de Belo Horizonte. Sistema de Informação Complementar.

nores de vinte anos nas regiões nordeste/norte, no extremo leste e extremo sul do município. A região central, equivalente aos bairros de melhores condições sócio-econômicas, apresenta conglomerados do tipo baixo-baixo.

Padrão muito semelhante ao anterior foi encontrado para escolaridade da mãe, representada na Figura 2. As regiões leste, nordeste e norte da cidade apresentaram conglomerados com altas proporções de mães com menos de oito anos de estudo (alto-alto), e a região central apresentou um conglomerado com baixas proporções de mães com baixa escolaridade (baixo-baixo).

Na Figura 3, encontra-se a distribuição dos conglomerados de mães primíparas. Neste mapa, observa-se a presença de um grande conglomerado com altas taxas de primíparas, cujos vizinhos também têm a mesma característica, ou seja, com taxas altas (alto-alto) na zona central do município, equivalentes aos distritos Centro-Sul, Leste, Noroeste e Pampulha. Observa-se, ainda, a presença de dois conglomerados de áreas do tipo baixo-baixo, um no Barreiro e outro, menor, no distrito Nordeste.

A distribuição de partos cesárea é apresentada na Figura 4. Essa figura segue o padrão da proporção de primíparas da Figura 3. Pode-se evidenciar um conglomerado tipo alto-alto na região central da cidade, e dois conglomerados tipo baixo-baixo, um em parte da região nordeste/norte e outro no extremo leste do município. Uma única área no extremo sul do município pode ser identificada como baixo-baixo.

A distribuição de conglomerados de áreas com mães com, pelo menos, um filho morto em gestações evidenciou dois conglomerados: um tipo baixo-baixo na região leste e outro tipo alto-alto no extremo sul, além de uma pequena área na região nordeste do município.

Quanto à proporção de mães com menos de quatro consultas pré-natais, pode-se evidenciar pequenos conglomerados do tipo alto-alto em parte das regiões nordeste e leste e uma pequena área no extremo sul, mostrando uma distribuição mais aleatória desse indicador quando comparado aos anteriores.

A distribuição da proporção de mães multíparas foi inversamente proporcional à de primíparas, e os indicadores prematuridade, asfixia moderada e grave no 1o e 5o minutos, baixo peso e muito baixo peso apresentaram padrão de distribuição mais aleatório, podendo-se evidenciar pequenas áreas de autocorrelação espacial local significativas.

A análise de correlação de Pearson mostrou associação linear positiva entre indicadores primíparas e cesárea $(r=0,725$, $\mathrm{p}<0,01)$; mães 
com baixa escolaridade e mães adolescentes ( $r=0,775, p<0,01)$, e com menos de quatro consultas no pré-natal $(r=0,643, p<0,01)$; e ainda, mães adolescentes e menos de quatro consultas no pré-natal $(\mathrm{r}=0,596, \mathrm{p}<0,01)$.

Correlações negativas foram consistentemente encontradas entre proporção de cesárea e menos de oito anos de estudo $(\mathrm{r}=-0,843, \mathrm{p}<0,01)$; cesárea e mães adolescentes $(r=-0,814, p<0,01)$; cesárea e filhos mortos $(r=-0,341, p<0,01)$; cesárea e menos de quatro consultas no pré-natal $(\mathrm{r}=-0,863, \mathrm{p}<0,01)$; primíparas e mães adolescentes $(r=-0,526, p<0,01)$; primíparas e menos de oito anos de estudo $(r=-0,731, p<0,01)$; e primíparas e menos de quatro consultas no pré-natal $(\mathrm{r}=0,525, \mathrm{p}<0,01)$.

Foi possível evidenciar três áreas de abrangência com autocorrelação espacial local significantes para quase todos os indicadores estudados. As proporções encontradas nessas três áreas, com algumas exceções, foram diferentes dos valores observados para o município, com padrão de taxas mais baixas para melhores indicadores e taxas mais altas para piores indicadores de saúde.

\section{Discussão}

Neste estudo, foi possível observar um padrão de distribuição espacial característico para alguns indicadores de precárias condições de saúde na gestação e parto das mães que tiveram seus filhos no ano de 2001, em Belo Horizonte. Destacam-se idade adolescente, baixa escolaridade, primiparidade, menor comparecimento ao pré-natal e maior proporção de filhos mortos em gestações anteriores. Três áreas de abrangência, em três diferentes regiões do município, configuraram-se como áreas que concentraram tais indicadores, cuja distribuição não aleatória guarda visível relação com as características sócio-demográficas, sendo possível evidenciar diferenciais sociais e de acesso aos serviços de saúde, identificando potenciais focos de pronta intervenção dentro dos distritos urbanos do município.

A análise utilizada nesta investigação demonstra que a combinação das informações obtidas através da vigilância em saúde com as técnicas de análises espaciais, uma vez mais, contribui para o entendimento da epidemiologia de agravos à saúde da mulher e da criança, sobretudo contextualizando geograficamente os eventos em áreas com baixas condições sócio-econômicas.

A grande variação geográfica encontrada na proporção de mães adolescentes, com áreas
Figura 1

Distribuição de conglomerados significantes de mães adolescentes. Belo Horizonte, Minas Gerais, Brasil, 2001*.

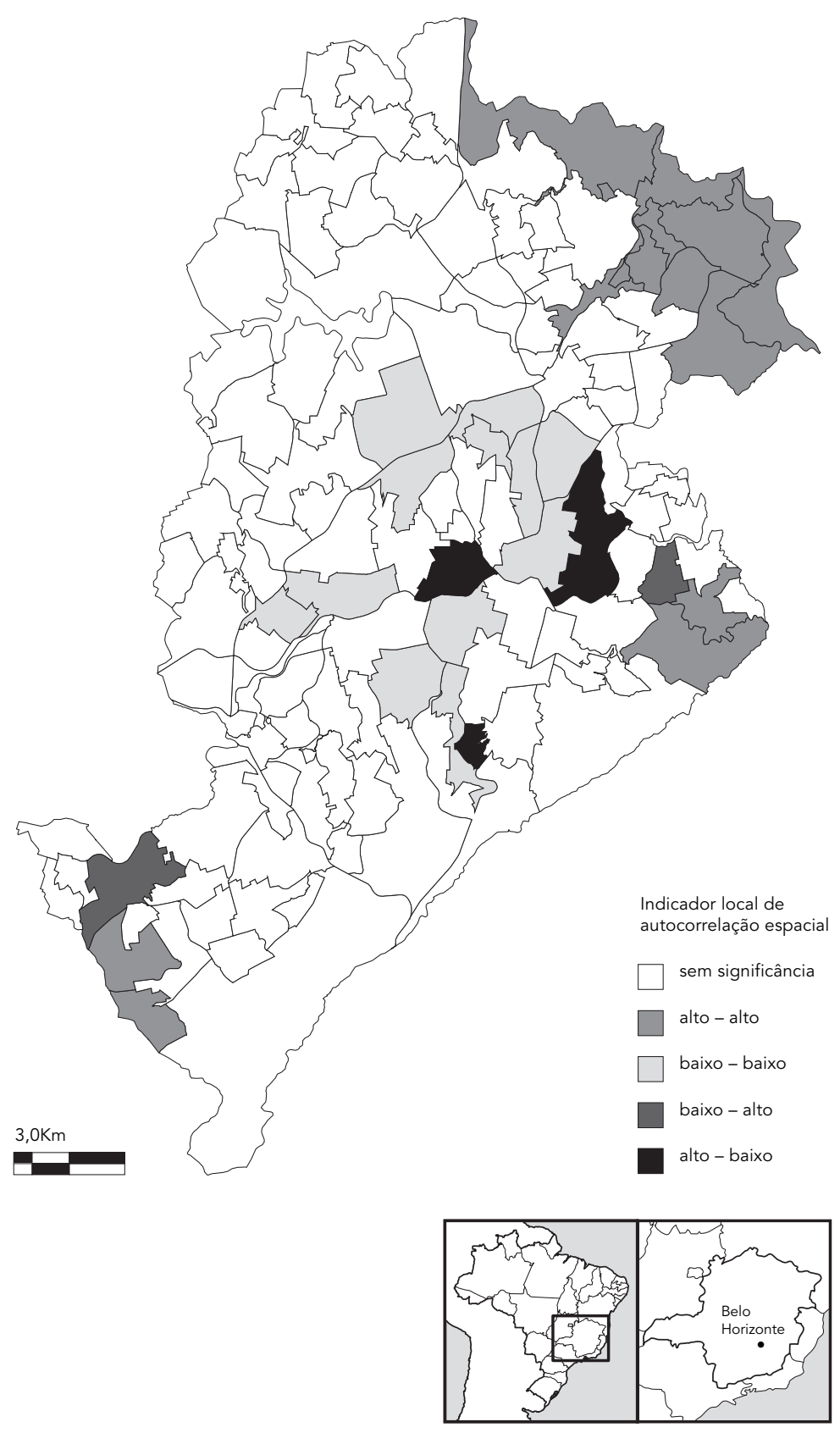

* Por área de abrangência das unidades básicas de saúde. 
Figura 2

Distribuição de conglomerados significantes de mães com $<8$ anos de estudo. Belo Horizonte, Minas Gerais, Brasil, 2001*

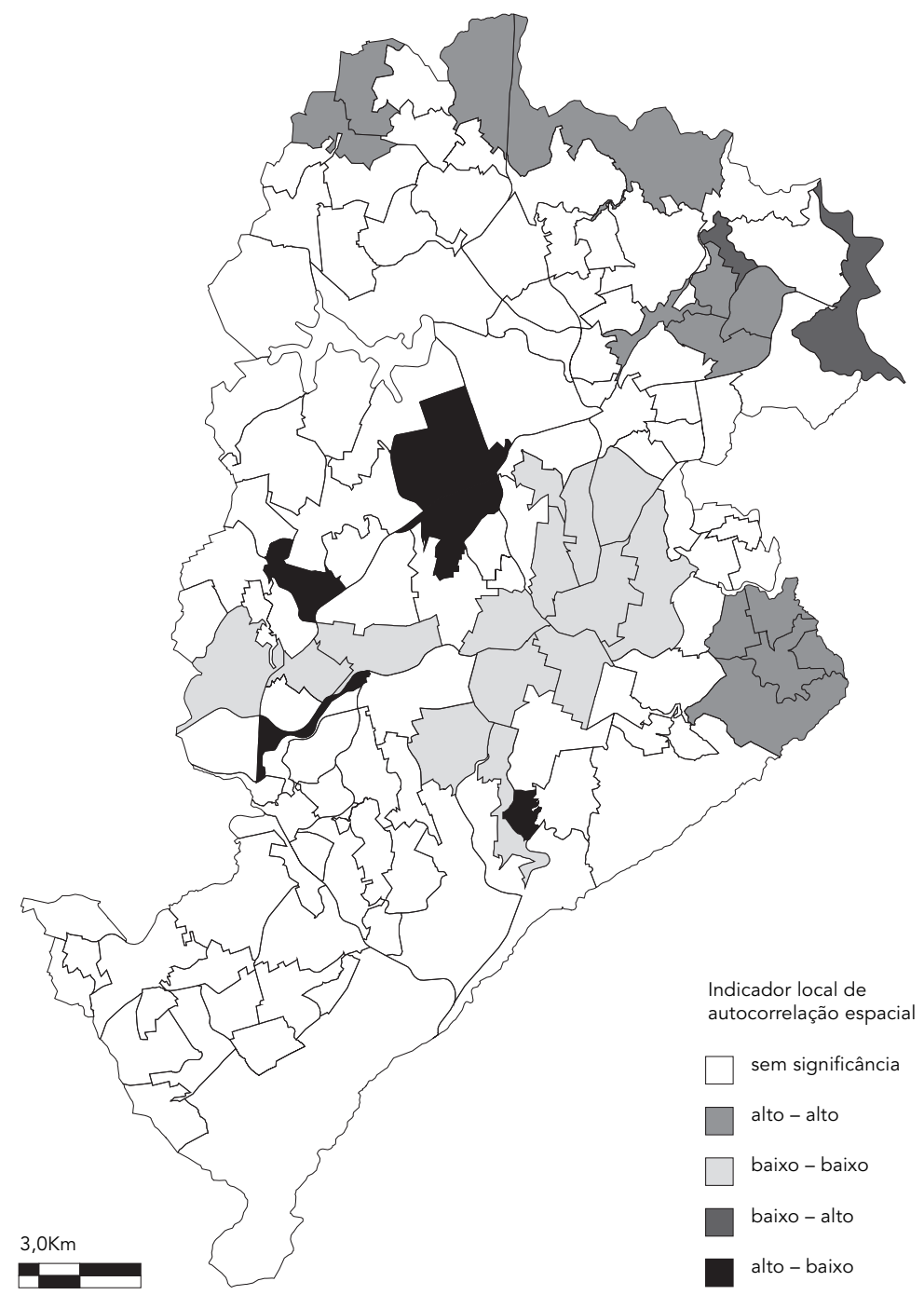

* Por área de abrangência das unidades básicas de saúde.

bem acima da média do município (16\%), a autocorrelação espacial global presente em distintas regiões do município e a presença de conglomerados de áreas com altas proporções de mães adolescentes em regiões com piores condições sócio-econômicas, notadamente em áreas próximas a favelas, representam uma evidência dessa associação. Reforçam essa associação as menores proporções de mães adolescentes encontradas em conglomerados de áreas de abrangência de bairros de classe média e al- ta. Corroboram, também, os achados de outros estudos, abordando a relação entre mães adolescentes e agravos no nascimento, como asfixia moderada e grave e maiores coeficientes de mortalidade neonatal, perinatal e infantil 1,5,6, 29,30. Outras evidências são os achados de gravidez na adolescência, em grupos sociais desfavorecidos 31,32 , e o crescimento das taxas de fecundidade nesse grupo, especialmente em camadas mais pobres da população 33 . No entanto, mais que fatores biológicos ligados às taxas de fecundidade, resultados negativos da gravidez na adolescência têm sido associados, recentemente, a influências sociais e ambientais 34. De fato, estudo conduzido no Município do Rio de Janeiro, por D’Orsi \& Carvalho 35 , mostrou maiores proporções de mães adolescentes em bairros com menor infra-estrutura e com padrão inverso ao da distribuição de cesáreas, bastante semelhante ao aqui encontrado, em que a correlação entre essas características foi de $81 \%$. Tais achados reforçam a relação entre gestação na adolescência e piores condições sócio-econômicas.

Quanto à proporção de mães com baixa escolaridade (média de 42,4\%), houve grande variação entre as áreas do município (10\% a $78,7 \%$ ), sugerindo diferenças significativas no acesso à educação. A autocorrelação espacial global e local mostrou padrões muito semelhantes ao de mães adolescentes, com altas proporções desse indicador em regiões de pior nível sócio-econômico, reforçado também pela alta correlação entre essas variáveis.

O grau de instrução materno tem sido entendido como um indicador da condição social relevante para a saúde dos filhos, influenciando fortemente não apenas o resultado da gestação, mas também a evolução das crianças 36 . Também, o maior grau de instrução da mãe tem sido descrito como facilitador do acesso ao trabalho e à melhoria da posição sócio-econômica da família 2. Segundo o Fundo das Nações Unidas para a Infância ${ }^{37}$, cada ano de estudo da mãe implica em redução da mortalidade infantil aproximadamente na ordem de 9 por mil. Além disso, a educação da mulher pode desempenhar papel importante na procura por serviços preventivos e na compreensão das implicações envolvidas na gravidez, parto e cuidados com o recém-nascido 34 .

Quanto à paridade da mãe, foram analisadas a distribuição de mães primíparas, com mais de três filhos nascidos vivos em gestações anteriores (multíparas), e aquelas com, pelo menos, um filho morto em gestações anteriores, ocorrendo, no município, respectivamente, em $41,9 \%$ e $5,1 \%$ para os dois últimos. 
Mães primíparas, ao contrário dos indicadores já discutidos, apresentaram inverso padrão espacial de autocorrelação global com baixa escolaridade e mães adolescentes. Observou-se que altas taxas de primíparas concentraram-se em regiões de classe média/alta (região central), e baixas taxas, nos bairros de piores condições sócio-econômicas. Alguns trabalhos têm mostrado associação entre primiparidade e baixo peso 4 , com maior renda familiar e melhores condições sócio-econômicas 31,32. Esse resultado é reforçado por nossos achados com respeito à multiparidade, que mostraram uma autocorrelação espacial global limítrofe ( $p=0,06$ ); e, na análise dos indicadores locais, foram detectadas duas pequenas áreas, uma da região leste e outra da norte (ambas próximas às favelas), que apresentavam conglomerados tipo alto-alto e uma área maior na região central (bairros de classe média) que apresentou menor proporção de mães com menos de quatro filhos. A multiparidade tem sido associada, em outros tipos de estudos não ecológicos, com o nível sócio-econômico e à morbimortalidade infantil 1,34,38.

Neste estudo, o padrão de distribuição espacial de mães com, pelo menos, um filho morto em gestações anteriores foi menos marcado. Maiores proporções concentraram-se no extremo sul do município e menores na região leste. Esse indicador é apontado como de risco para baixo peso, retardo do crescimento intra-uterino e para mortalidade infantil, fetal e neonatal, além de associar-se à baixa renda familiar 1,3,30 .

Padrões bastante evidentes foram aqueles relativos aos partos cesárea, com proporções variando entre $28 \%$ e $67 \%$, entre as áreas de abrangência. A autocorrelação espacial global e a análise de indicadores locais mostraram um padrão bastante característico, com conglomerados de áreas com altas taxas de cesáreas nas regiões de melhores condições sócio-econômicas e de proporções baixas de cesáreas em regiões menos favorecidas, sugerindo fortemente uma associação entre cesárea e nível sócioeconômico alto. É sabido que a prática da cesariana, um reconhecido avanço da medicina, teve, a partir da década de 70, um crescimento indiscriminado em vários países 39. Entre os países da América Latina, o Brasil ocupa o segundo lugar, ultrapassando, em muito, o preconizado pela Organização Mundial da Saúde (OMS) 40 , que propõe taxa máxima de $15 \%$ de cesariana para qualquer país. Alguns estudos têm demonstrado associação positiva entre as taxas de cesáreas e padrão sócio-econômico, com elevada proporção de cesariana entre mulheres com maior escolaridade e renda, e me-
Figura 3

Distribuição de conglomerados significantes de mães primíparas. Belo Horizonte, Minas Gerais, Brasil, 2001*.

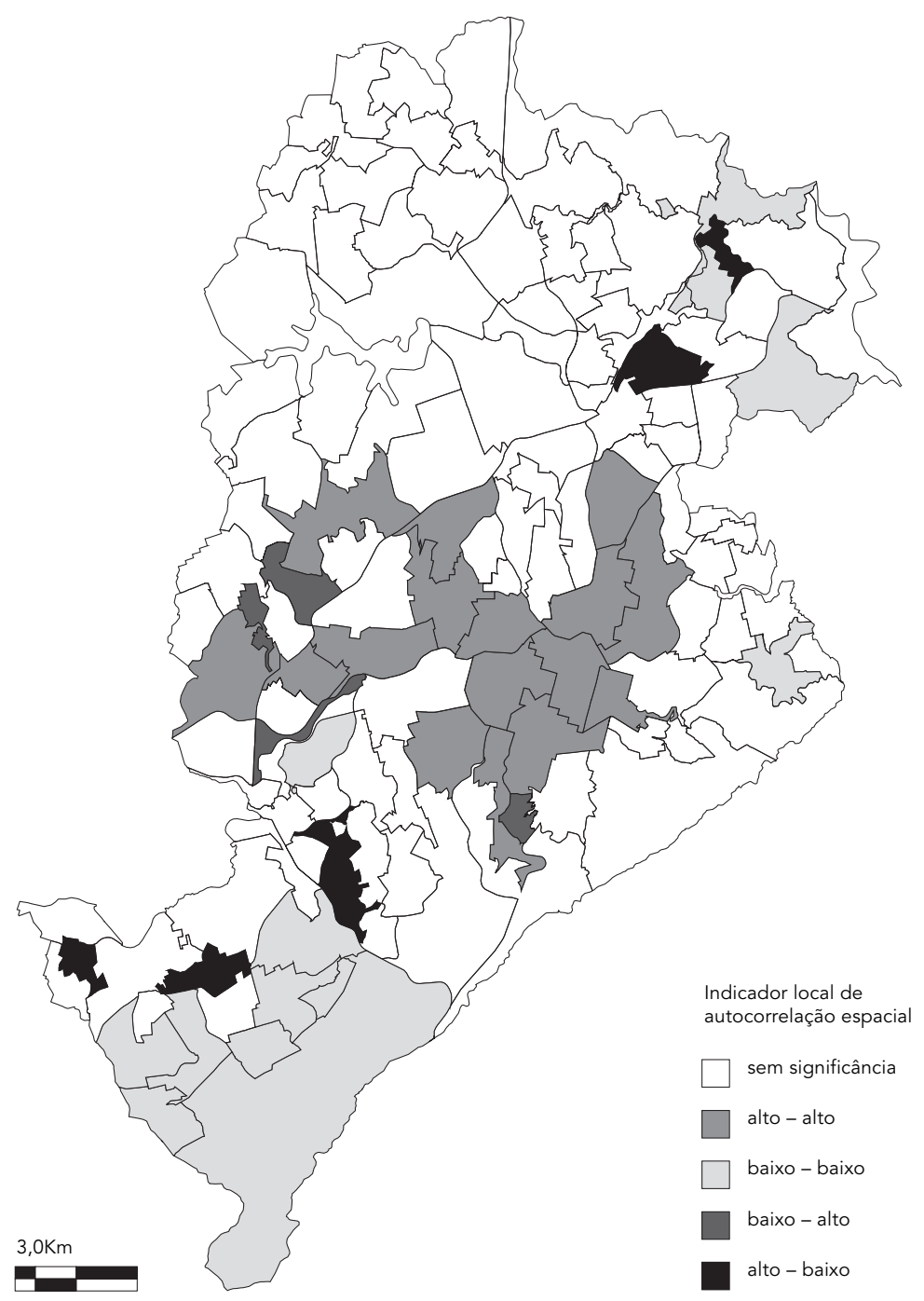

* Por área de abrangência das unidades básicas de saúde.

nores proporções dessa prática em mães adolescentes 10,41. Além disso, Oliveira \& Leal 42 encontraram uma taxa média de cesáreas de $38,3 \%$ para o Estado do Rio de Janeiro, sendo $67,5 \%$ em maternidades conveniadas. Também um estudo da distribuição espacial dos nascimentos no Município do Rio de Janeiro mostrou valores mais elevados de cesáreas nos bairros de melhores condições sócio-econômicas 35. Essas investigações corroboram os achados deste nosso estudo, sugerindo que essa as- 
Figura 4

Distribuição de conglomerados significantes de cesáreas.

Belo Horizonte, Minas Gerais, Brasil, 2001*

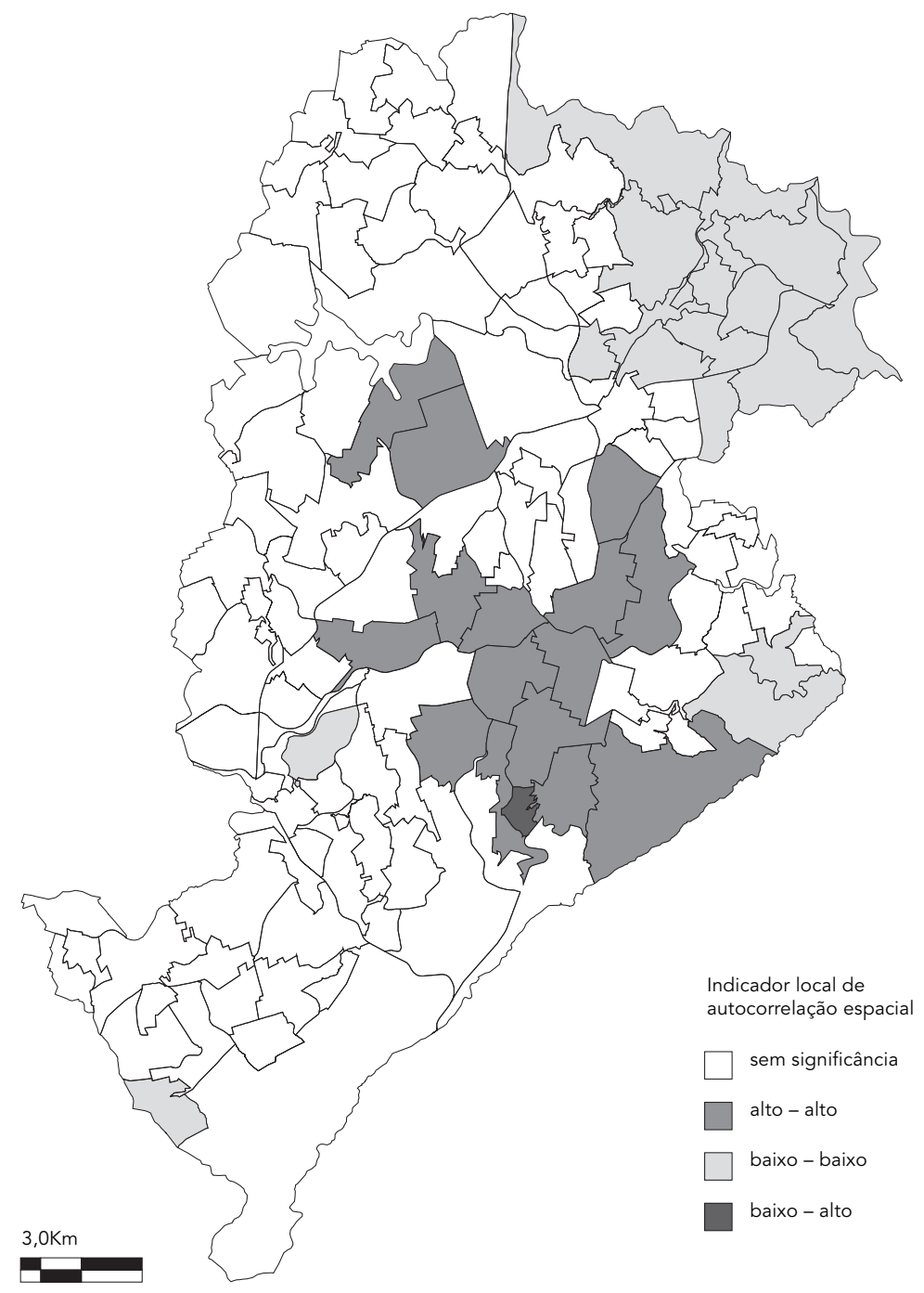

* Por área de abrangência das unidades básicas de saúde.

sociação representa importante marcador do nível sócio-econômico.

Neste estudo, apesar da boa cobertura prénatal, com aproximadamente $88 \%$ das mães tendo realizado mais de quatro consultas, cerca de $10 \%$ das mães fizeram menos de quatro consultas no pré-natal, com variação entre $4 \%$ e $17,8 \%$. A distribuição espacial foi não aleatória, evidenciando dois conglomerados do tipo altoalto em áreas próximas às favelas, sugerindo condições ainda precárias em algumas localida- des, de acordo com o novo modelo de assistência pré-natal proposto pela OMS, que preconiza o mínimo de quatro consultas no pré-natal como uma medida de qualidade ${ }^{43}$. Além do mais, é conhecido que o baixo número de consultas no pré-natal tem sido associado à morbimortalidade materna e infantil e às condições sócioeconômicas desfavoráveis, como baixa escolaridade e baixa renda familiar 8,30,41,44.

Quanto às características do recém-nascido, a maioria dos indicadores apresentou baixa ou nenhuma autocorrelação significativa. Apesar de alguns estudos encontrarem associações de prematuridade, asfixia, baixo e muito baixo peso com nível sócio-econômico, neste estudo, salvo a presença de alguns poucos conglomerados merecedores de uma investigação detalhada de fatores associados, não foi detectado nenhum padrão espacial típico para esses indicadores.

Baixo peso e prematuridade são apontados como fortes indicadores da situação sócio-econômica e social das populações, além de serem fortemente associados à morbimortalidade e representarem os fatores isolados mais importantes na determinação da sobrevivência infantil, como encontrado em vários estudos 1,45,46,47. Entretanto, tratando-se de distribuição espacial, os achados têm sido contraditórios. D'Orsi \& Carvalho 35 encontraram distribuição espacial aleatória do baixo peso na cidade do Rio de Janeiro, similar a este estudo. Já Victora et al. 48 encontraram diferenças significativas no padrão de distribuição espacial do baixo peso no Estado do Rio Grande do Sul, estando a ocorrência desse associada às áreas de piores condições sócio-econômicas. Também Morenoff 14 , em estudo sobre a distribuição espacial do baixo peso realizado na cidade de Chicago, encontrou associação com áreas de ocorrência de crimes violentos, ressaltando o componente sócioeconômico desse indicador. Ressalta-se que ocorrência de baixo peso é excessiva $(10,6 \%)$, maior inclusive que a prematuridade $(7,7 \%)$, sugerindo um componente de retardo do crescimento intra-uterino nesse indicador que, embora relacionado às condições sócio-econômicas adversas, não apresentou padrão espacial característico neste estudo.

O que parece consistente, no entanto, é a ausência de padrões espaciais das características ligadas ao recém-nascido, sugerindo que tais características não foram detectadas pelas determinações espaciais tanto quanto o foram as características maternas e do atendimento pré e perinatal. Portanto, os indicadores que melhor caracterizaram as áreas de abrangência e as regiões do Município de Belo Horizonte fo- 
ram mães adolescentes, menos de oito anos de estudo, ser primípara, ter filhos mortos em gestações anteriores, parto cesárea e ter menos de quatro consultas no pré-natal. Podemos observar uma tendência de concentração de áreas com melhores indicadores nas áreas de abrangência da região central da cidade e conglomerados com indicadores desfavoráveis em três regiões da cidade: extremo sul (parte do Barreiro), leste e nordeste. Nessas regiões, três áreas de abrangência se destacaram como áreas que apresentaram taxas altas de mães adolescentes; mães com menos de oito anos de estudo; mães que fizeram menos de quatro consultas no pré-natal e que tiveram, pelo menos, um filho nascido morto em gestações anteriores; acompanhadas de taxas baixas de cesariana e de primíparas. Esses indicadores são altamente relacionados entre si e sugestivos de diferenças sócio-econômicas, como apontado pela análise de correlação de Pearson.

Várias considerações merecem ser ressaltadas antes de uma conclusão final. Tem-se discutido que a utilidade de dados secundários para análises espaciais depende da qualidade da informação. Os dados aqui utilizados parecem ter uma boa qualidade, como apontado em estudo anterior que, além de mostrar uma boa cobertura do sistema de notificação dos nascidos vivos, mostrou qualidade e confiabilidade das informações encontradas no SINASC 22.

Outra discussão que se apresenta refere-se à utilização de áreas de abrangência e não de setores censitários como unidades de estudo, pois os primeiros poderiam ser insuficientes para detectar diferenças importantes intraáreas. A possível alternativa da utilização de unidades menores, como os setores censitá- rios, tem introduzido instabilidade nas estimativas das taxas, devido à flutuação aleatória, mesmo com uso da estimativa Bayesiana empírica 13 , ou a análise de dados pontuais, considerando o local de residência de cada recémnascido, agregados por setores censitários. Um outro argumento refere-se à manutenção da divisão administrativa dos serviços de saúde, facilitando possíveis e futuras intervenções.

Apesar dos limites expostos acima, este estudo, de base comunitária, conduzido para testar a metodologia de análise espacial, buscando indicadores de risco para os nascimentos em uma área delimitada, demonstrou que essa metodologia configurou-se como um ótimo instrumento de detecção de conglomerados de áreas de risco à saúde materno infantil. Esse instrumento de análise pode ser incorporado como mecanismo de monitoramento dos eventos relacionados aos nascimentos no Município de Belo Horizonte, uma vez que possibilita a priorização de intervenções e elaboração de estratégias educativas, preventivas e assistenciais em áreas específicas e, ao mesmo tempo, constitui-se uma ferramenta simples com utilização de dados qualificados e acessíveis. Novos estudos que abordem associações com outras variáveis medidas no nível coletivo poderão contribuir sobremaneira para a melhor elucidação das relações entre fatores sócio-econômicos e saúde materno infantil. Os achados deste estudo apontam para a necessidade de vigilância contínua, especialmente nas regiões destacadas, e de promoção de intervenções educativas e de assistência adequada, seja para melhorar o acesso aos serviços, seja para discutir práticas obstétricas bastante consolidadas.

\section{Resumo}

A análise espacial de indicadores de saúde tem sido um importante instrumento na detecção de diferenciais intra-urbanos. O estudo objetivou traçar um perfil dos nascimentos em Belo Horizonte, Minas Gerais, Brasil, em 2001, analisando a presença de conglomerados espaciais de indicadores de saúde do recém-nascido e suas mães, a partir de dados do Sistema de Informações sobre Nascidos Vivos. Para cada área de abrangência das Unidades Básicas de Saúde, foram calculadas as proporções desses indicadores, utilizando-se o método Bayesiano empírico. Para análise espacial, foram utilizados os índices de Moran global e local (LISA). Áreas com índices de autocorrelação espacial significantes $(p \leq 0,05)$ foram visualizadas através de mapas. Foram encontrados conglomerados de áreas com índices de autocorrelação espacial significativos para os indicadores adolescentes, menos de oito anos de estudo, filhos mortos em gestações anteriores, cesárea e menos de quatro consultas no pré-natal, guardando relação com as características sócio-demográficas das áreas. A metodologia utilizada configurou-se como um ótimo instrumento de detecção de conglomerados de áreas de risco à saúde materno infantil, podendo facilmente ser incorporada como mecanismo de monitoramento dos eventos relacionados aos nascimentos em Belo Horizonte.

Análise Espacial; Indicadores de Saúde; Saúde Materno-infantil; Condições Sociais 


\section{Colaboradores}

A. A. L. Friche e W. T. Caiaffa conceberam, delinearam e escreveram o manuscrito. M. C. M. Almeida e C. C. César colaboraram na análise estatística e discussão dos dados e, junto com L. M. F. Goulart, revisaram criticamente o conteúdo do artigo. Todos os autores revisaram e aprovaram o manuscrito final.

\section{Referências}

1. Victora CG, Barros FC, Vaughan JP. Epidemiologia da desigualdade. 2a Ed. São Paulo: Editora Hucitec; 1989.

2. Rodrigues CS, Magalhaes Jr. HM, Evangelista PA, Ladeira RM, Laudares S. Perfil dos nascidos vivos no Município de Belo Horizonte, 1992-1994. Cad Saúde Pública 1997; 13:53-7.

3. Menezes AMB, Barros FC, Victora CG, Tomasi E, Halpern R, Oliveira ALB. Fatores de risco para mortalidade perinatal em Pelotas, RS, 1993. Rev Saúde Pública 1998; 32:209-16.

4. Costa CE, Gotlieb SLD. Estudo epidemiológico do peso ao nascer a partir da declaração de nascido vivo. Rev Saúde Pública 1998; 32:328-34.

5. Bohland AK, Mello-Jorge MHP. Mortalidade de menores de um ano de idade na região do sudoeste do Estado de São Paulo. Rev Saúde Pública 1999; 33:366-73.

6. Silva AAM, Coimbra LC, Silva RA, Alves MTSSB, Lamy Filho F, Lamy ZC, et al. Perinatal health and mother-child health care in the municipality of São Luís, Maranhão State, Brazil. Cad Saúde Pública 2001; 17:1412-23.

7. Lansky S, França E, Leal MC. Mortes perinatais evitáveis em Belo Horizonte, Minas Gerais, Brasil, 1999. Cad Saúde Pública 2002; 18:1389-400.

8. Coimbra LC, Silva AAM, Mochel EG, Alves MTSSB, Ribeiro VS, Aragão VMF, et al. Fatores associados à inadequação do uso da assistência pré-natal. Rev Saúde Pública 2003; 37:456-62.

9. Malta DC, Almeida MCM, Dias MAS, Mery EE. A mortalidade infantil em Belo Horizonte, Minas Gerais, Brasil, por área de abrangência dos Centros de Saúde (1994-1996). Cad Saúde Pública 2001; 17:1189-98.

10. Campos TP. Perfil de nascimentos e óbitos infantis: a busca da assistência no Município do Rio de Janeiro [Dissertação de Mestrado]. Rio de Janeiro: Escola Nacional de Saúde Pública, Fundação Oswaldo Cruz; 1997.

11. Morais Neto OL, Barros MBA, Martelli CMT, Silva SA, Cavenaghi SM, Siqueira Jr. JB. Diferenças no padrão de ocorrência da mortalidade neonatal e pós-neonatal no Município de Goiânia, Brasil, 1992-1996: análise espacial para identificação das áreas de risco. Cad Saúde Pública 2001; 17:124150 .

12. Câmara G, Monteiro AM. Técnicas de geocomputação para análise espacial: é o caso para dados de saúde? Cad Saúde Pública 2001; 17:1059-71.

\section{Agradecimentos}

Este trabalho foi desenvolvido no Programa de Pósgraduação em Saúde Pública da Faculdade de Medicina da Universidade Federal de Minas Gerais como parte dos requisitos para obtenção do título de mestre em Saúde Pública.
13. Carvalho MS, Cruz OG. Análise espacial por microáreas: métodos e experiências. In: Veras RP, Barreto MB, Almeida Filho N, Barata RB, organizadores. Epidemiologia: contextos e pluralidade. Rio de Janeiro: Editora Fiocruz; 1998. p. 79-89.

14. Morenoff JD. Neighborhood mechanisms and the spatial dynamics of birth weight. Am J Sociol 2003; 108:976-1017.

15. Clarke KC, McLaffery SL, Tempalski BJ. On epidemiology and geographic information systems: a review and discussion of future directions. Emerg Infect Dis 1996; 2:85-92.

16. Croner CM, Sperling J, Broome FR. Geographic information systems (GIS): new perspectives in understanding human health and environmental relationship. Stat Med 1996; 15:1961-77.

17. Leal MC, Szwarcwald CL. Características da mortalidade neonatal no Estado do Rio de Janeiro na década de 80: uma visão espaço-temporal. Rev Saúde Pública 1997; 31:457-65.

18. Moore DA, Carpenter TE. Spatial analytical methods and geographic information systems: use in health research and epidemiology. Epidemiol Rev 1999; 21:143-61.

19. Machado Coelho GL, Assunção R, Mayrink W, Caiaffa WT. American cutaneous leishmaniasis in Southeast Brazil: space-time clustering. Int J Epidemiol 1999; 28:982-9.

20. Dias MAS, Caiaffa WT, Machado-Coelho GL. Internações e reinternações por Asma no Município de Belo Horizonte, MG, Brasil, 1997 a 2000: apontando um caminho. In: VII Congresso Brasileiro de Saúde Coletiva, Resumos. Brasília: Associação Brasileira de Pós-graduação em Saúde Coletiva; 2003. p. 53.

21. Almeida MCM, Caiaffa WT, Assunção RM, Proietti FA. Dinâmica intra-urbana das epidemias de Dengue em Belo Horizonte, MG, 1996-2002. In: VI Congresso Brasileiro de Epidemiologia. Recife: ABRASCO; 2004.

22. Friche AAL, Caiaffa WT, César CC, Goulart LMF. Qualidade dos dados do Sistema de Informação sobre Nascidos Vivos - SINASC - em Belo Horizonte, 1999. In: VI Congresso Brasileiro de Epidemiologia, 2004. Recife: ABRASCO; 2004.

23. Secretaria Municipal de Saúde de Belo Horizonte. Organização territorial dos serviços municipais de saúde em Belo Horizonte. Belo Horizonte: Grupo de Trabalho de Territorialização, Secretaria Municipal de Saúde de Belo Horizonte; 2003. 
24. Assunção RM, Barreto SM, Guerra HL, Sakurai E. Mapas de taxas epidemiológicas: uma abordagem Bayesiana. Cad Saúde Pública 1998; 14:71323.

25. Bailey TC, Gatrell AC. Interactive spatial data analysis. Essex: Longman Scientific \& Technical; 1995.

26. Anselin L. Local indicators of spatial association - LISA. Geogr Anal 1995; 27:91-115.

27. Anselin L, Syabri I, Smirnov O. Visualizing multivariate spatial correlation with dynamically linked windows. Urbana-Champaign: Spatial Analysis Laboratory, Department of Agricultural and Consumer Economics, University of Illinois; 2004.

28. Hanson CE, Wieczoreck WF. Alcohol mortality: a comparison of spatial clustering methods. Soc Sci Med 2002; 55:791-802.

29. Gama SGN, Szwarcwald CL, Leal MC, Theme-Filha MM. Gravidez na adolescência como fator de risco para baixo peso ao nascer no Município do Rio de Janeiro, 1996 a 1998. Rev Saúde Pública 2001; 35:74-80.

30. Almeida MF, Novaes HMD, Alencar GP, Rodrigues LC. Mortalidade neonatal no Município de São Paulo: influência do peso ao nascer e de fatores sócio-demográficos e assistenciais. Rev Bras Epidemiol 2002; 5:93-107.

31. Tomasi E, Barros FC, Victora CG. As mães e suas gestações: comparação de duas coortes de base populacional no Sul do Brasil. Cad Saúde Pública 1996; 12 Suppl 1:21-5.

32. Martins C, Almeida MF. Fecundidade e diferenciais intra-urbanos de desenvolvimento humano, São Paulo, Brasil, 1997. Rev Saúde Pública 2001; 35:421-7.

33. Gama SGN, Szwarcwald CL, Leal MC. Experiência de gravidez na adolescência, fatores associados e resultados perinatais entre puérperas de baixa renda. Cad Saúde Pública 2002; 18:153-61.

34. Bale JR, Stoll BJ, Lucas AO. Improving birth outcomes. Meeting the challenge in developing world. Washington DC: Committee on Improving Birth Outcomes, Institute of Medicine, National Academy of Sciences; 2003.

35. D'Orsi E, Carvalho MS. Perfil de nascimentos no Município do Rio de Janeiro: uma análise espacial. Cad Saúde Pública 1998; 14:367-79.

36. Goulart LMHF, Xavier CC, Goulart EMA, Somarriba MG, Almeida CG, Costa ALDG. Avaliação da ação de vigilância à morbimortalidade infantil na periferia de Belo Horizonte, Minas Gerais. Rev Bras Saúde Mater-Infant 2003; 3:291-304.
37. Fundo das Nações Unidas para a Infância. Situação mundial da infância. Brasília: Fundo das Nações Unidas para a Infância; 1990.

38. Mello-Jorge MHP, Gotlieb SLD, Soboli MLMS, Almeida MF, Latorre MRD. Avaliação do sistema de informação sobre nascidos vivos e o uso de seus dados em epidemiologia e estatísticas de saúde. Rev Saúde Pública 1993; 27 Suppl:1-46.

39. Faundes A, Ceccatti JG. Operação cesárea no Brasil. Incidência, tendências, causas, conseqüências e propostas de ação. Cad Saúde Pública 1991; 7:150-73.

40. World Health Organization. Care in normal birth; a practical guide, maternal and newborn health/ safe motherhood unit. Family and reproductive health. Geneva: World Health Organization; 1996.

41. Costa JSD, Victora CG, Barros FC, Halpern R, Horta BL, Manzolli P. Assistência médica materno-infantil em duas coortes de base populacional no Sul do Brasil: tendências e diferenciais. Cad Saúde Pública 1996; 12 Suppl 1:59-6.

42. Oliveira MIC, Leal MC. Alojamento conjunto e parto cesáreo em maternidades do Estado do Rio de Janeiro, Brasil. Rev Saúde Pública 1997; 31: 572-80.

43. World Health Organization. Ensayo clínico aleatorizado de control prenatal de la OMS: manual para la puesta en práctica del nuevo modelo de control prenatal. Geneva: Department of Reproductive Health and Research, World Health Organization; 2003.

44. Zambonato AMK, Pinheiro RT, Horta BL, Tomasi E. Fatores de risco para nascimento de crianças pequenas para idade gestacional. Rev Saúde Pública 2004; 38:24-9.

45. Horta BL, Barros FC, Halpern R, Victora CG. Baixo peso ao nascer em duas coortes de base populacional no Sul do Brasil. Cad Saúde Pública 1996; 12 Suppl 1:27-31.

46. Accioly MC. Determinantes da mortalidade neonatal em Belo Horizonte (1993): subsídios para o planejamento da atenção materno-infantil [Dissertação de Mestrado]. Belo Horizonte: Faculdade de Medicina, Universidade Federal de Minas Gerais; 1997.

47. Mariotoni GGB, Barros FAA. Peso ao nascer e mortalidade hospitalar entre nascidos vivos, 19751996. Rev Saúde Pública 2000; 34:71-6.

48. Victora C, Grassi PR, Schmidt AM. Situação da saúde da criança em área da região Sul do Brasil, 1980-1982: tendências temporais e distribuição espacial. Rev Saúde Pública 1994; 28:423-32.

Recebido em 04/Mai/2005

Versão final reapresentada em 29/Set/2005

Aprovado em 08/Nov/2005 